\title{
Effects of peptides and amino acids derived from oyster on blood lipids
}

\begin{abstract}
Objectives: The objective of this study is to demonstrate the effect on blood lipids in humans, of a supplement which contained peptides and amino acids originating from oyster meat.

Methods: Proteins in oyster meat were hydrolyzed by enzymes, followed by preparing oyster meat extract powder containing the peptides and free amino acids as a supplement. We performed the following 2 clinical trials in which male and female adults with dyslipidemia received the oyster meat supplement for specified periods; in the first open label trial, the oyster meat supplement was given to 14 subjects (average age 48.7 years old) for 8 weeks, while in the second crossover controlled trial, the oyster meat supplement and its placebo were taken by 19 subjects (average age 52.4years old) for 4weeks, respectively. In these trials, the effect of the supplement on blood lipids was evaluated by conducting serum chemistry and body composition measurement before and after taking the supplement
\end{abstract}

Results: In the open label trial, at the end of Week 4 from the start of taking the supplement, a decrease of very low-density lipoprotein (VLDL) and an increase of high-density lipoproteins (HDL) in blood lipoproteins were observed with statistically significant difference $(\mathrm{P}<0.01)$.

In the crossover placebo-controlled study, we confirmed the reduction of the ratio of VLDL in lipoproteins $(\mathrm{P}=0.04)$, the increase of HDL cholesterol $(\mathrm{P}=0.02)$, and the suppression of the elevation of triglycerides (TG) $(\mathrm{P}=0.02)$ Week 4after the start of taking of the supplement.

Conclusion: The oyster meat extract powder was most likely to have potential utilities in the management of dyslipidemia.

Keywords: oyster meat, amino acids, peptides, blood lipids
Volume I Issue 5 - 2014

\author{
Masaru Ohtani,' Taizo Seki, ${ }^{2,3}$ Masahiko \\ Ooe, ${ }^{4,5}$ Yuichiro Itoh, ${ }^{6}$ Yasuyuki Okamoto ${ }^{6}$ \\ 'Graduate of School of Frontier Sciences, The University of \\ Tokyo, Japan \\ ${ }^{2}$ Graduate School of Bioagricultural Sciences, Nagoya University, \\ Japan \\ ${ }^{3}$ Noevir Co Ltd., R\&D, Japan \\ ${ }^{4}$ Graduate School of Medicine, Osaka University, Japan \\ ${ }^{5}$ TOKIWA Pharmaceutical Co., Ltd, Japan \\ ${ }^{6}$ Sansei Hospital, Japan
}

Correspondence: Masaru Ohtani, Sports Sciences for Health and Activity, Graduate School of Frontier Sciences, The University of Tokyo, 5-I-5 Kashiwanoha, Kashiwa-shi, Chiba-ken 277-8563, Tel +8I047|364644, FAX, 047|364644,

Email ohtani@k.u-tokyo.ac.jp

Received: September 5, 2014 | Published: October 13, 2014
Abbreviations: ALB, albumin; ALT, alanineamino transferase; AST, aspartate aminotransferase; BMI, body mass index; CETP, cholesteryl ester transfer protein; CRE, creatinine; G6PDH, glucose-6-phospho dehydrogenase; $\gamma$-GT, $\gamma$-glutamyl transpeptidase; HDL, high-density lipoprotein; HDL-C, high density lipoprotein cholesterol; hs-CRP, high sensitivity c-reactive protein; LDL, low density lipoprotein; LDL-C, low density lipoprotein cholesterol; SREBP, sterol regulatory element-binding protein; TG, triglyceride; Total-C, total cholesterol; TP, total protein; UA, uric acid; UN, urea nitrogen; VLDL, very low-density lipoprotein; JHFA, japan health food authorization

\section{Introduction}

Japan is one of the countries with the longest healthy life expectancy, however, arteriosclerotic disease has been still one of the major causes of death of Japanese, while the death rate due to this disease is low in Japan compared with Europe and America. Therefore, it is required to overcome arteriosclerotic disease including cardiovascular and cerebrovascular disorders, which respectively rank the second and third causes of Japanese death, in order to attain further improvement of Japanese life span with better quality of life. We think that the lifestyle habits largely affect the development of arteriosclerotic diseases; because dyslipidemia, hypertension, diabetes, and smoking have been defined as the risk factors of arteriosclerotic diseases. Japanese diets have become westernized and the incidence of dyslipidemia in Japan has become not much different from that in western countries. As a result, the control of dyslipidemia has become important for the prevention of arteriosclerotic diseases. ${ }^{1}$ Excessive or deficient state of blood lipids is categorized into the following 3 types:
i. High LDL-C (low density lipoprotein cholesterol) type
ii. Low HDL-C (high density lipoprotein cholesterol) type
iii. High TG (high Triglyceride )type

For these 3 types, standard values have been respectively determined, and a person whose blood concentration of lipids is over the standard value is diagnosed as dyslipidemia. Since there are no subjective symptoms in patients with dyslipidemia, the control of dyslipidemia has been achieved by regular tests and health guidance. In the Japanese Atherosclerosis Guideline 2012, the upper borderline of hyper-LDL-cholesterolemia expressed in terms of LDL-C value is defined to be 120 to $139 \mathrm{mg} / \mathrm{dL}^{2}$ Oyster (Crassostrea gigas) is important aquatic resources for Japanese, and it has a long history as food in the world. In terms of the quantity of production, Japan is the third, following China and Korea. ${ }^{3}$ Since oyster meat abundantly contains proteins, Taurine, glycogen, and zinc as important nutrients. In Japan, it has been used as a raw material of a supplement in addition to its popularity as seafood. 
To extract the components useful for health from oysters, we have used a hydrothermal process in general. In addition, there is another process in which proteins of oyster meat are hydrolyzed by a protease, thereby producing oyster meat extract powder which contains a large quantity of free amino acids and peptides. In this connection, we have previously reported the effects of amino acids on physiological functions including nutritional improvements, muscle fatigue reduction, and immune system improvement. ${ }^{4-7}$ In Japan, attention has been paid to investigations on functional foods which have focused on the nutrition and functions of peptides and amino acids obtained by decomposing proteins in recent years.

At least part of peptides orally taken can be absorbed in the body as such, and transferred to systemic blood flow, followed by exerting functionality. For example, a TG-lowering effect in blood is reported with respect to tetra-peptides obtained by the enzyme digestion of haemoglobin. ${ }^{8}$ There are reports that specific peptides degraded from oyster proteins have a hypotensive effect and antiHIV activity associated with the angiotensin converting-enzyme inhibition and its application to HIV patients has been anticipated. ${ }^{9}{ }^{10}$ Effects of oyster meat extract powder on blood lipid improvement have been recently verified by using an animal model. ${ }^{11}$ Thus, the increase of serum cholesterol levels in rats that had been fed with cholesterol-loading feed was suppressed by the intake of feed mixed with the oyster meat extract powder. On the other hand, there are not many studies on the blood lipid-improving effects in humans by the functional foods of oyster meat extracts. In this study, a supplement was prepared by blending oyster meat powder, and with medical workers as subjects, 2 tests were performed on male and female adults who were suspected lipid abnormality by health examinations. First, a preliminary clinical trial was carried out by the open label design, and the changes of blood indices by the continuous intake of the oyster meal extract powder supplement for 8 weeks were examined. Next, to demonstrate the effect of a supplement containing peptides and amino acids derived from oyster meat on blood lipids, we performed the placebo controlled clinical trial in which male and female adults with dyslipidemia received the oyster meat supplement for 4 weeks.

Table I Demographic characteristics, serum chemistry data for screening, and food and nutrient consumption before start of studies

Table IA Open label study $(n=14)$

\begin{tabular}{|c|c|c|c|c|}
\hline & $\begin{array}{l}\text { Paragraph } \\
\text { Determined }\end{array}$ & $\begin{array}{l}\text { Measurement } \\
\text { Values(Mean } \pm \text { SEM) }\end{array}$ & $\begin{array}{l}\text { No. of subjects deviating } \\
\text { from standard value }\end{array}$ & $\begin{array}{l}\text { Standard } \\
\text { value }\end{array}$ \\
\hline \multirow{5}{*}{$\begin{array}{l}\text { Demographic } \\
\text { Characteristics }\end{array}$} & Age, y & $48.7 \pm 7.5$ & - & - \\
\hline & Male & 10 & - & - \\
\hline & Female & 4 & - & - \\
\hline & Weight, kg & $70.5 \pm 2.9$ & - & - \\
\hline & $\begin{array}{l}\text { Body mass index, } \\
\mathrm{kg} / \mathrm{m}^{2}\end{array}$ & $25.3 \pm 0.7$ & 6 & $>25 \mathrm{~kg} / \mathrm{m}^{2}$ \\
\hline \multirow{3}{*}{ Serum Chemistry Data } & LDL-C, mg/dL & $145.5 \pm 11.9$ & 7 & $>140 \mathrm{mg} / \mathrm{dL}$ \\
\hline & $\mathrm{HDL}-\mathrm{C}, \mathrm{mg} / \mathrm{dL}$ & $50.5 \pm 3.3$ & 3 & $<40 \mathrm{mg} / \mathrm{dL}$ \\
\hline & $\mathrm{TG}, \mathrm{mg} / \mathrm{dL}$ & $241.6 \pm 43.3$ & 8 & $>150 \mathrm{mg} / \mathrm{dL}$ \\
\hline
\end{tabular}

Table I B Randomized, single-blind, crossover, placebo-controlled comparative study $(n=19)$

\begin{tabular}{|c|c|c|c|c|}
\hline & $\begin{array}{l}\text { Paragraph } \\
\text { determined }\end{array}$ & $\begin{array}{l}\text { Measurement } \\
\text { values(Mean } \pm \text { SEM) }\end{array}$ & $\begin{array}{l}\text { No. of subjects } \\
\text { deviating from } \\
\text { standard value }\end{array}$ & Standard value \\
\hline \multirow{5}{*}{$\begin{array}{l}\text { Demographic } \\
\text { Characteristics }\end{array}$} & Age, $y$ & $52.4 \pm 7.9$ & - & - \\
\hline & Male & 9 & - & - \\
\hline & Female & 10 & - & - \\
\hline & Weight, kg & $65.6 \pm 2.8$ & - & - \\
\hline & $\begin{array}{l}\text { Body mass index, } \\
\mathrm{kg} / \mathrm{m}^{2}\end{array}$ & $24.4 \pm 0.9$ & 7 & $>25$ \\
\hline \multirow{3}{*}{ Serum Chemistry Data } & LDL-C, mg/dL & $|5| .8 \pm 27 . \mid$ & 13 & $>140$ \\
\hline & HDL-C, mg/dL & $59.4 \pm 18.5$ & 2 & $<40$ \\
\hline & $\mathrm{TG}, \mathrm{mg} / \mathrm{dL}$ & $151.5 \pm 99.7$ & 9 & $>150$ \\
\hline
\end{tabular}




\begin{tabular}{|c|c|c|c|c|}
\hline & $\begin{array}{l}\text { Paragraph } \\
\text { determined }\end{array}$ & $\begin{array}{l}\text { Measurement } \\
\text { values(Mean } \pm \text { SEM) }\end{array}$ & $\begin{array}{l}\text { No. of subjects } \\
\text { deviating from } \\
\text { standard value }\end{array}$ & Standard value \\
\hline \multirow{6}{*}{ Nutrients* } & Energy, kcal & $2233.7 \pm 155$ & 9 & $\begin{array}{l}<2450 \text { (Male), } \\
<1950 \text { (Female) }\end{array}$ \\
\hline & Protein, $\mathrm{g}$ & $79.0 \pm 5.0$ & 19 & $<60$ \\
\hline & Fat, $g$ & $50.6 \pm 2.9$ & 19 & $>20,<25$ \\
\hline & \multirow[t]{2}{*}{ Carbohydrate, $\mathrm{g}$} & \multirow[t]{2}{*}{$297.8 \pm 24.3$} & \multirow[t]{2}{*}{19} & $>50,<70$ \\
\hline & & & & $<9$ (Male), \\
\hline & Salt, g & $12.8 \pm 0.6$ & 19 & $<7.5$ (Female) \\
\hline
\end{tabular}

*Nutrition standard values are those of Japanese 50's generation. LDL-C, low density lipoprotein cholesterol; HDL-C, high density lipoprotein cholesterol;TG, triglyceride; SEM, standard error of means

\section{Materials and methods}

The study was reviewed and approved by the ethical review committees of Sansei Hospital in Kobe before starting the trials in the hospital. A preliminary open label trial was carried out from May to July 2009, while a major single blind crossover placebo-controlled study from April to July 2011.

Healthcare professionals in Sansei Hospital were recruited as subjects. Male and female adults with dyslipidemia whose demographic data, serum chemistry data, and nutrients are shown in Table 1 were selected according to the data of serum chemistry (TG $>149 \mathrm{mg} / \mathrm{dL}$ or LDL-C $>139 \mathrm{mg} / \mathrm{dL}$ or HDL-C $<40 \mathrm{mg} / \mathrm{dL}$ ). In the open label study, 14 subjects consisting of 10 males and 4 females whose mean age was $48.7 \pm 7.5$ years old were selected. In the crossover placebo-controlled study, 19 subjects consisting of 9 males and 10 females whose mean age was $52.4 \pm 7.9$ years old were selected. We confirmed that the subjects were not in medical treatment at the time of inclusion by a questionnaire, surveyed the lifestyle habits such as the quantity of meal, drinking, smoking, the exercise frequency before the trials, and instructed them not to change their lifestyle habits throughout the study period. This trial was performed pursuant to the Declaration of Helsinki, and we provided sufficient and adequate explanations to the subjects as to the followings; the test objectives and methods: anticipated interests and benefits and risks: if subjects do not agree to the study participation, it does not cause any disadvantage to said subjects: even after agreeing to participate, subjects can withdraw his or her agreement at any time: and personal information of the subjects is to be protected. Consents in writing by free will were obtained from the subjects.

\section{Preparation of test supplement, administration method, and administration duration}

The test supplements used in this study conform to the standard of the oyster health foods defined by the Japan Health Food Authorization (JHFA) and were produced by the method described by Tanaka et al., ${ }^{11}$ (manufactured by Tensei Suisan Co., Ltd.). The oyster meat extract powder was made into tablets, followed by sugar coating. The tablets were used as the test supplement. The placebo tablets whose color and size were the same as those of the test supplements were prepared by replacing the oyster meat extract powder to sugar alcohols.

The subjects were administered 6 test supplement tablets or 6 placebo tablets twice a day (b.i.d.) in the morning and evening after meals (total 12 tablets per day). Each test tablet used in the open label trial contained $200 \mathrm{mg}$ of oyster meat extract powder, totaling $2,400 \mathrm{mg}$ in the 12-tablets administered per day. The nutritional contents were as follows: $20 \mathrm{kcal}$ of energy, $1.0 \mathrm{~g}$ of proteins, $0.1 \mathrm{~g}$ of fats, and $4.0 \mathrm{~g}$ of carbohydrates. The analysis of the protein fraction by a highperformance liquid chromatography method shows that the protein fraction in the 12 tablets was composed of approximately $590 \mathrm{mg}$ of peptides and approximately $170 \mathrm{mg}$ of free amino acids. That is, the daily dose (in 12 tablets) contained approximately $750 \mathrm{mg}$ of peptides and free amino acids derived from the oyster meat in the open label trial. An analysis using a size exclusion liquid chromatography method confirmed that the peptides whose molecular weights were $500 \mathrm{Da}$ or less accounted for about $60 \%$ of the entire peptides. The enzymatic hydrolysis conditions for the preparation of the oyster meat extract powder used in the crossover placebo-controlled study were changed from that of the open label study according to the procedure reported by Tanaka et al., ${ }^{11}$ and thereby the peptide content was increased (Table 2). Although the peptide content in the oyster meat extract powder was increased by changing the hydrolysis conditions, the amount of peptides in a supplement tablet used in the placebo-controlled study was adjusted to $180 \mathrm{mg}$ which was similar to that in the supplement tablet used in the open label study. Accordingly, the tablets used in the 2 clinical trials were comparable. In 12 tablets administered per day, totally $2,160 \mathrm{mg}$ of oyster meat extract powder was contained together with the nutritional contents of $21 \mathrm{kcal}$ of energy, $1.0 \mathrm{~g}$ of proteins, $0.1 \mathrm{~g}$ of lipids, and $4.0 \mathrm{~g}$ of carbohydrates. The protein fraction in the 12 tablets was composed of approximately $650 \mathrm{mg}$ of peptides and approximately $130 \mathrm{mg}$ of free amino acids. As a result, each subject took approximately $780 \mathrm{mg}$ of peptides and free amino acids derived from oyster meat in a day in the placebo controlled trial. The peptides whose molecular weights were $500 \mathrm{Da}$ or less accounted for about $70 \%$ of the entire peptides in the tablet.

In the open label study, the subjects were to receive the supplement tablets sequentially for 8 weeks, and serum chemistry was performed at Week 4 and Week 8 . In the single blind crossover placebocontrolled study, we set a dormant duration of 4weeks between the administration of the oyster supplement tablets and the placebo tablets for 4weeks, respectively (Figure 1). The subjects were unable to distinguish the placebo tablets from the supplement tablets. The order to take the supplement tablets and placebo tablets was randomly allocated to each subject. 
Table 2 Ingredients, nutrients, and functional elements in oyster extract powder contained in 12 supplement tablets given daily, and molecular weight distribution of peptides in oyster extract powders

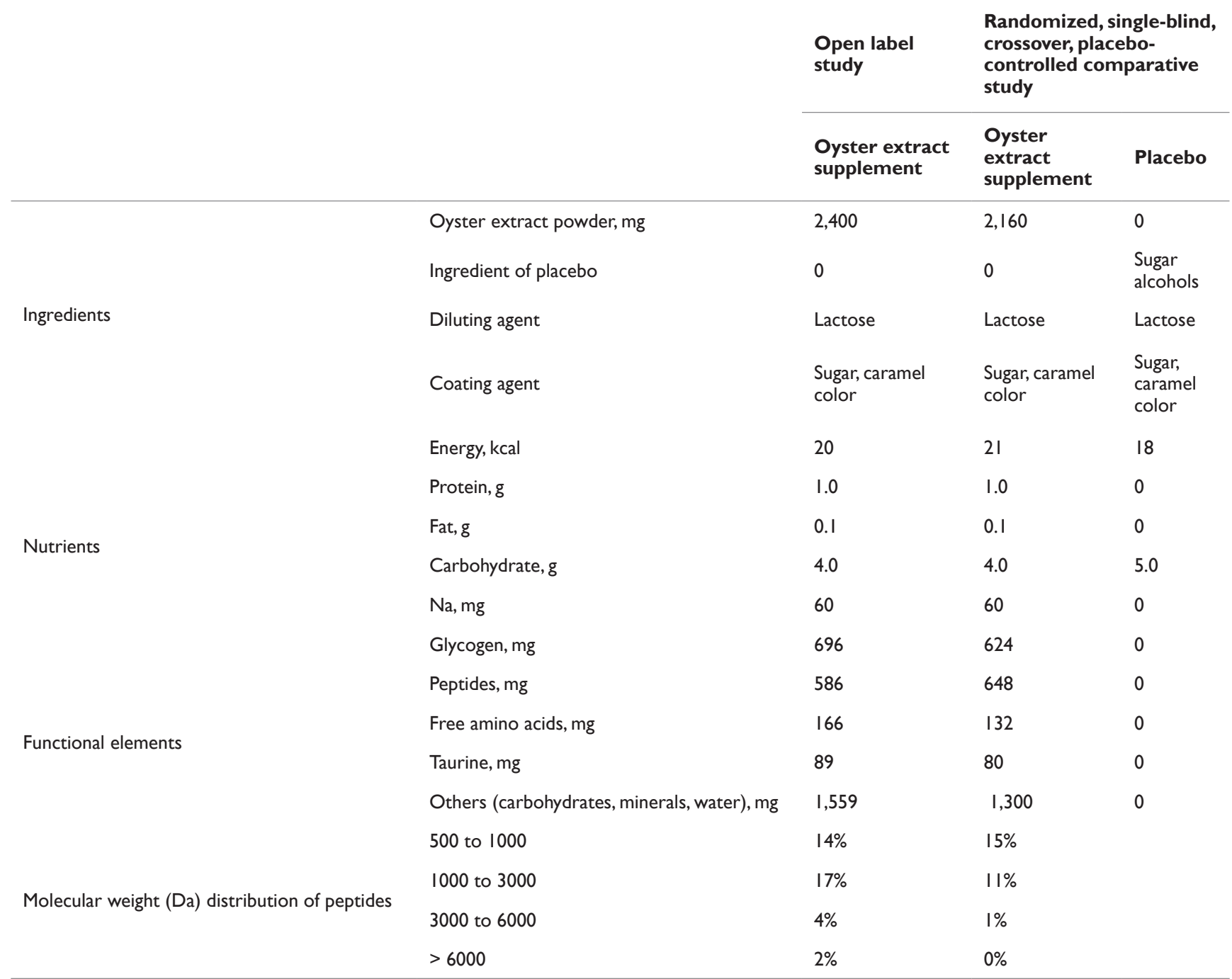

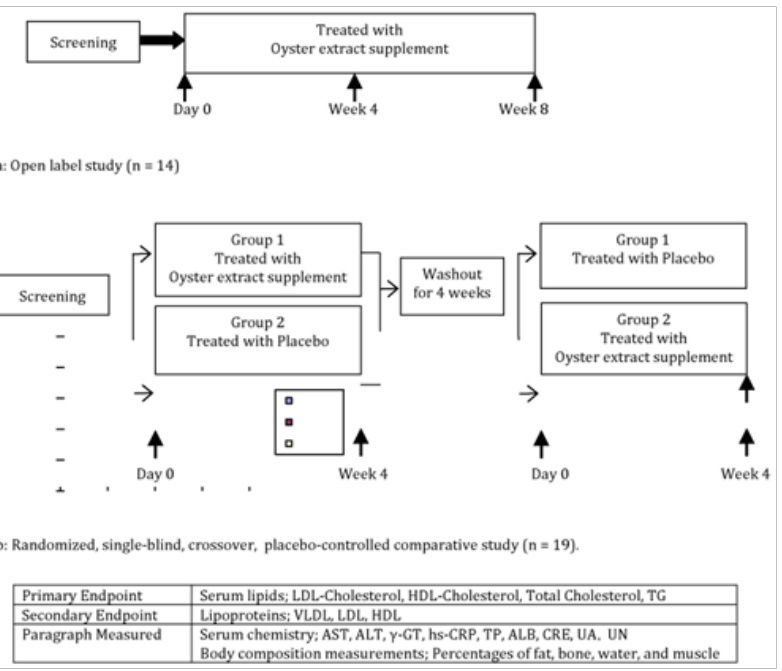

Figure I Clinical study design. 


\section{Measurement of serum chemistry values}

From the subjects fasting from 21:00 of the day before the measurement day, blood was collected at 9:00 in the morning of the measurement day, and the following paragraphs were measured: AST, ALT, $\gamma$-GT, LDL-C, HDL-C, total cholesterol, TG, hs-CRP, total proteins (TP), albumin (ALB), creatinine (CRE), uric acid (UA), and urea nitrogen (UR). Here, the concentrations of LDL-C, HDL-C, total cholesterol, and TG were determined by the enzyme methods. A precision measurement was adopted for lipoprotein fractions; chylomicrons (VLDL, IDL, LDL, and HDL) were analyzed by a polyacrylamide gel disc electrophoresis by which the chylomicrons were separated in this order according to the particle sizes. These analyses were performed by Mitsubishi Chemical Medience Co., Ltd.

\section{Body composition measurement}

Body weight, lean body mass, body water mass, fat mass, muscle mass, skeletal muscle mass, mass of in-organics, protein mass, body mass index (BMI), body fat percentage, body fat mass, amount of visceral fat, and mass of subcutaneous fat were measured by the bioelectrical impedance analysis method, using body components measuring devices (X-SCAN Plus professional, Jawon Medical Co., Ltd.).

\section{Methods of statistical analyses and entry method of numerical values}

The data were statistically analyzed by using SPSS (PASW) Statistics 18 (IBM Japan, Ltd.). In the open label trial, the measured values at the beginning of the study, Week 4 , and Week 8 were pursued. Analysis of variance was performed for the differences of the mean values at the 3 points, and the repeated-measure analysis of variance (ANOVA) or Friedman test was adopted. A multiple comparison was made for the differences of the mean values at each point, and Bonferroni correction or Steel-Dwass test was adopted. In the crossover placebo-controlled study, the measured values at the beginning of the study and Week 4 were pursued. The paired t-test was performed for the difference of the average values at each point, while regarding the inter group difference of the average values, the t-test or Mann-Whitney U test was used on the assumption of the normal distribution of population. When the significance level was less than $5 \%$, we judged there was a significant difference, and in case where the level was less than $10 \%$, we judged there was a tendency. Values were denoted as Mean $\pm \mathrm{SEM}$.

\section{Results}

\section{Serum chemistry values}

Demographic characteristics, serum chemistry data, and nutrients of each subject are shown in Table 1. There were no large difference for the demographic data including age, BMI, and weight between the two trials. Among 14 subjects included in the open label trial, 7 subjects showed high LDL-C, 3 subjects low HDL-C, and 8 subjects high TG (there were overlaps) in comparison with the standard values of Japanese patients with dyslipidemia. Among 19 subjects included in the crossover placebo-controlled trial, 13 subjects showed high LDL-C, 2 subjects low HDL-C, and 9 subjects high TG (there were overlaps). All the subjects developed one or more lipid abnormalities. At the time of screening, there was no subject with hyperglycemia which is one of the factors affecting a lipid profile.

\section{Serum chemistry values in open label trial}

Serum chemistry values examined in the open label trial are presented in Table 3. The ANOVA was performed for the differences of the mean values at the 3 points (before administration, Week 4 and Week 8 after the initiation of administration) in the group taking the supplement tablets; as a result, LDL-C of blood lipid tended to decrease at Week $8(\mathrm{P}=0.09)$.In the multiple comparison test, no significant change was observed at Week 4 and Week 8 in comparison with the values before the treatment with the supplement tablets. Although the change of an HDL-C value was slight, the ANOVA indicates that there was a significant reduction in this parameter $(\mathrm{P}=0.02)$, whereas HDL increased significantly $(\mathrm{P}=0.006)$. The ANOVA demonstrates the significant decrease of VLDL $(\mathrm{P}=0.006)$. In the next step, we performed the ANOVA for the lipoprotein fractions by sex; the result showing significant reduction of VLDL at Week 4 and Week 8 in male subjects with $\mathrm{P}=0.02(\mathrm{n}=10)$, but not in female subjects (Figure 2). In the multiple comparison test on the VLDL mean values of male subjects, no significant difference was observed among the 3 points. Regarding the values of VLDL of female subjects, no significant difference was indicated probably due to the small number of the subjects, but VLDL was decreased as was observed in male subjects. The changes of other parameters of serum chemistry were small, however, the TP, HDL-C, LDL-C, and ALB decreased temporally at Week 4, and recovered at Week 8. These Bell-shape changes indicate the likely presence of a placebo effect or changes in eating habits and/or exercise habits of subjects during the first 4-weeks. The decrease of serum HDL-C contradicted the increase of HDL, though the reason was not obvious. Although the placebo effect was suggested, since regarding VLDL, LDL, and HDL determined by the precision measurement of lipoprotein fractions, there was no large difference between Week 4 and Week 8 and the decrease of VLDL and HDL at these 2 time points were statistically significant $(\mathrm{P}=0.006)$ in comparison with the data before administration, we planned to perform a crossover placebo-controlled study in the next step, so as to eliminate the placebo effect if there is and to especially confirm the effect of oyster meat supplement on HDL and HDL-C.

\section{Serum chemistry values in crossover placebo- controlled trial}

Measured values in the supplement and the placebo groups are summarized in Table 4, and no significant changes were observed in most of the parameters. In Table 4, the parameters whose standard values are different between male and female subjects were analyzed by sex. There were no paragraphs which indicated sex-dependent differences. The significant difference was observed in HDPLC and VLDL between the supplement group and placebo group; thus, only in the supplement group HDL-C increased significantly $(\mathrm{P}=0.04)$, while VLDL reduced $(\mathrm{P}=0.03)$.

$\%$ Change of each parameter was examined. As shown in Figure $3,{ }^{1} \mathrm{HDL}-\mathrm{C}$ of the supplement group increased significantly compared with that of the placebo group ( $\mathrm{P}=0.02)$. TG in the placebo group increased at Week 4 temporally, while TG in the supplement group did not increase or was significantly suppressed as shown in Figure $3(\mathrm{P}=0.02) .{ }^{2}$ In addition, Figure $3^{3}$ indicates the significant decrease of VLDL in the supplement group $(\mathrm{P}=0.04)$ as observed in the open label study. 
Table 3 Changes of serum chemistry parameters at Weeks 4 and 8 after initiation of administration of oyster extract supplement from those before administration in open label study

\begin{tabular}{|c|c|c|c|c|c|c|}
\hline & Unit & Standard & Initial & 4Weeks & 8Weeks & PaMean \pm SEM \\
\hline HDL-C & $\mathrm{mg} / \mathrm{dL}$ & $\begin{array}{l}m 40-85 \\
\text { f 40-95 }\end{array}$ & $50.5 \pm 3.3$ & $46.8 \pm 3.0$ & $50.1 \pm 2.8$ & $0.02 *$ \\
\hline LDL-C & $\mathrm{mg} / \mathrm{dL}$ & $65-139$ & $145.5 \pm 1 \mid .9$ & $135.6 \pm 10.5$ & $|4| .6 \pm 1 \mid .4$ & 0.09 \\
\hline Total-C & $\mathrm{mg} / \mathrm{dL}$ & $120-219$ & $244.7 \pm 12.7$ & $231.1 \pm 9.1$ & $234.3 \pm 10.9$ & 0.11 \\
\hline TG & $\mathrm{mg} / \mathrm{dL}$ & $30-149$ & $241.6 \pm 43.3$ & $252.4 \pm 45.3$ & $228.9 \pm 33.0$ & 0.65 \\
\hline VLDL & $\%$ & $\begin{array}{l}m 5-20 \\
\text { f } 4-17\end{array}$ & $18.8 \pm 1.6$ & $|5.| \pm \mid .3$ & $15.3 \pm 1.5$ & $0.006 * *$ \\
\hline LDL & $\%$ & $\begin{array}{l}m \text { 44-69 } \\
\text { f 42-65 }\end{array}$ & $45.2 \pm 3.1$ & $46.4 \pm 2.5$ & $46.9 \pm 2.5$ & 0.67 \\
\hline HDL & $\%$ & $\begin{array}{l}m 22-50 \\
\text { f } 26-53\end{array}$ & $18.8 \pm 1.0$ & $21.9 \pm 1.1$ & $20.4 \pm 1.0$ & $0.006 * *$ \\
\hline AST & IU/L & $10-40$ & $26.4 \pm 3.5$ & $22.7 \pm 2.1$ & $21.6 \pm 1.5$ & 0.27 \\
\hline ALT & IU/L & $5-45$ & $39.9 \pm 10.2$ & $25.4 \pm 2.6$ & $25.1 \pm 2.7$ & 0.16 \\
\hline$\gamma-\mathrm{GT}$ & IU/L & $\begin{array}{l}\mathrm{m} 80 \\
\mathrm{f} 30\end{array}$ & $63.1 \pm 12.8$ & $55.4 \pm 8.6$ & $56.7 \pm 9.5$ & 0.39 \\
\hline TP & $\mathrm{g} / \mathrm{dL}$ & $6.7-8.3$ & $7.6 \pm 0.1$ & $7.4 \pm 0.1$ & $7.5 \pm 0.1$ & $0.03 *$ \\
\hline ALB & $d / d L$ & $3.8-5.3$ & $4.7 \pm 0.1$ & $4.6 \pm 0.1$ & $4.7 \pm 0.1$ & 0.57 \\
\hline hs-CRP & $\mathrm{mg} / \mathrm{dL}$ & 0.3 or less & $0.12 \pm 0.04$ & $0.07 \pm 0.02$ & $0.08 \pm 0.02$ & 0.19 \\
\hline CRE & $\mathrm{mg} / \mathrm{dL}$ & $0.61-1.04$ & $0.8 \pm 0.0$ & $0.8 \pm 0.0$ & $0.8 \pm 0.0$ & 0.25 \\
\hline UA & $\mathrm{mg} / \mathrm{dL}$ & $3.8-7.0$ & $5.8 \pm 0.2$ & $5.6 \pm 0.2$ & $5.7 \pm 0.2$ & 0.35 \\
\hline UN & $\mathrm{mg} / \mathrm{dL}$ & $8-20$ & $13.6 \pm 0.6$ & $14.0 \pm 0.8$ & $\mid 4.1 \pm 0.6$ & 0.82 \\
\hline
\end{tabular}

$\mathrm{P}^{\mathrm{a}}$ values were calculated using the repeated measure analysis of variance (ANOVA).VLDL, LDL, and HDL were evaluated using the Friedman test; $*,<0.05$; **, < 0.0I; m, Male; f, Female; ALB, albumin, ALT, alanine aminotransferase;AST, aspartate aminotransferase; CRE, creatinine, $\square$-GT, $\square$-glutamyl transpeptidase; HDL, high-density lipoprotein; HDL-C, high density lipoprotein cholesterol; hs-CRP, high sensitivity c- reactive protein; LDL, low density lipoprotein; LDL-C, low density lipoprotein cholesterol;TG, triglyceride; total-C, total cholesterol,TP, total protein; UA, uric acid; UN, urea nitrogen;VLDL, very low-density lipoprotein; SEM, standard error of means

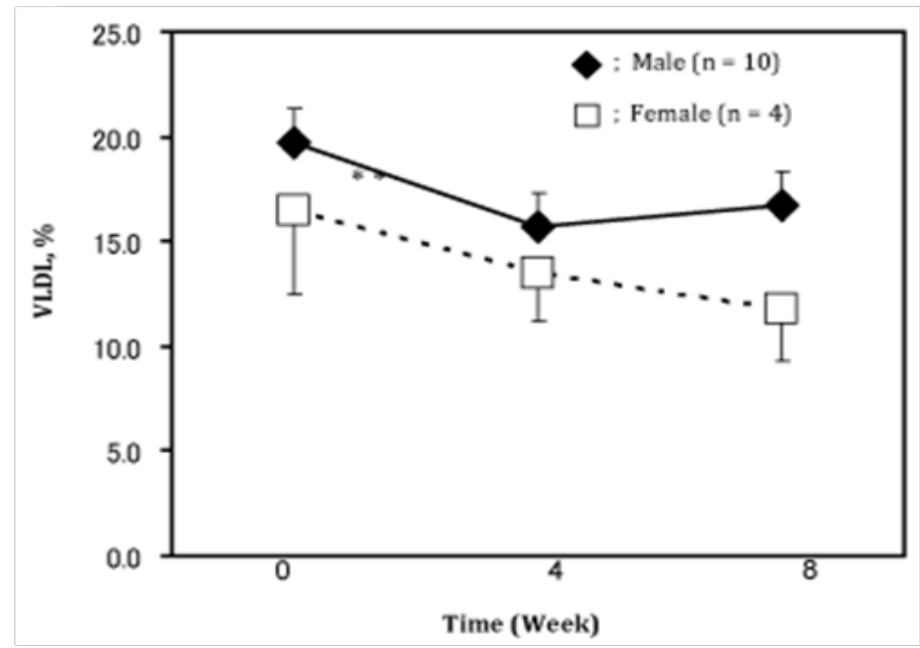

Figure 2 Changes of the means of \%VLDL over time in each male and female group supplemented with the oyster extract in the open label study. Points, mean value; Bars, SEM; *, significantly different from the value before administration with $\mathrm{P}=0.02$ (Friedman test) 
Table 4 Changes due to oyster extract supplemented in the serum biochemical profile and body compositions prior to and following the dietary study period in the crossover, placebo-controlled comparative study

Table 4A Serum chemistry data

\begin{tabular}{|c|c|c|c|c|c|c|c|c|c|}
\hline \multirow[b]{2}{*}{$\begin{array}{l}\text { Serum } \\
\text { chemistry } \\
\text { data }\end{array}$} & \multirow[b]{2}{*}{ Unit } & \multirow[b]{2}{*}{ Standard } & \multirow[b]{2}{*}{ Sex } & \multicolumn{2}{|c|}{ Before dosing } & \multicolumn{2}{|c|}{ Final(Week 4) } & \multicolumn{2}{|c|}{ PaMean $\pm S E M$} \\
\hline & & & & Active $^{b}$ & Placebo & Active $^{b}$ & Placebo & $\begin{array}{l}\text { Active; } \\
\text { initial vs } \\
\text { final }\end{array}$ & $\begin{array}{l}\text { Final; } \\
\text { active vs } \\
\text { placebo }\end{array}$ \\
\hline \multicolumn{10}{|c|}{ Serum chemistry data } \\
\hline & & $m$ 40-85 & $\mathrm{m}$ & $44.7 \pm 3.6$ & $46.89 \pm 2.2$ & $48.22 \pm 3.0$ & $46.8 \pm 3.6$ & 0.09 & 0.26 \\
\hline \multirow[t]{2}{*}{ HDL-C } & $\mathrm{mg} / \mathrm{dL}$ & f $40-95$ & $f$ & $66.8 \pm 4.8$ & $71.00 \pm 5.2$ & $68.50 \pm 4.8$ & $68.4 \pm 5.3$ & 0.30 & 0.95 \\
\hline & & & & $56.3 \pm 3.9$ & $59.60 \pm 4.0$ & $58.90 \pm 3.7$ & $58.2 \pm 4.1$ & $0.04 *$ & 0.48 \\
\hline LDL-C & $\mathrm{mg} / \mathrm{dL}$ & $65-139$ & & $149.3 \pm 6.3$ & $156.10 \pm 6.1$ & $154.10 \pm 6.4$ & $153.9 \pm 5.3$ & 0.11 & 0.97 \\
\hline Total-C & $\mathrm{mg} / \mathrm{dL}$ & $120-219$ & & $239.5 \pm 6.6$ & $245.70 \pm 6.9$ & $239.30 \pm 6.5$ & $242.3 \pm 5.3$ & 0.95 & 0.54 \\
\hline \multirow[t]{2}{*}{ TG } & $\mathrm{mg} / \mathrm{dL}$ & $30-149$ & & $165.3 \pm 21.3$ & $130.30 \pm 35.6$ & $\mid 47.80 \pm 18.5$ & $|7| .7 \pm 29.0$ & 0.18 & 0.17 \\
\hline & & m 5-20 & $\mathrm{m}$ & $15.3 \pm 1.6$ & $12.78 \pm \mid .4$ & $13.44 \pm 6.15$ & $12.7 \pm 1.6$ & 0.25 & 0.81 \\
\hline \multirow[t]{3}{*}{ VLDL } & $\%$ & $f 4-17$ & $f$ & $10.3 \pm 1.0$ & $9.30 \pm 1.0$ & $8.10 \pm 0.9$ & $10.3 \pm 1.1$ & 0.08 & 0.35 \\
\hline & & & & $12.7 \pm 1.1$ & $10.90 \pm 0.9$ & $10.60 \pm 1.3$ & $11.4 \pm 1.0$ & $0.03 *$ & 0.15 \\
\hline & & $m$ 44-69 & $\mathrm{m}$ & $50.6 \pm 1.3$ & $53.8 \pm 2.9$ & $51.9 \pm 2.5$ & $52.7 \pm 2.1$ & 0.37 & 0.73 \\
\hline \multirow[t]{3}{*}{ LDL } & $\%$ & f 42-65 & $f$ & $58.10 \pm 2.4$ & $57.2 \pm 1.8$ & $58.5 \pm 1.8$ & $55.0 \pm 2.1$ & 0.83 & 0.24 \\
\hline & & & & $54.5 \pm 1.6$ & $55.6 \pm 1.7$ & $55.4 \pm 1.6$ & $53.9 \pm 1.5$ & 0.46 & 0.27 \\
\hline & & m 22-50 & $\mathrm{m}$ & $18.6 \pm 1.4$ & $19.9 \pm 1.4$ & $18.9 \pm 1.1$ & $20.3 \pm 1.2$ & 0.59 & 0.05 \\
\hline \multirow[t]{2}{*}{ HDL } & $\%$ & f $26-53$ & $f$ & $24.5 \pm 1.5$ & $25.7 \pm 1.5$ & $24.8 \pm 1.6$ & $25.7 \pm 1.7$ & 0.35 & 0.31 \\
\hline & & & & $21.5 \pm 1.2$ & $23.0 \pm 1.2$ & $22.0 \pm 1.2$ & $23.2 \pm 1.22$ & 0.29 & 0.06 \\
\hline AST & IU/L & $10-40$ & & $22.3 \pm 1.6$ & $23.0 \pm 1.4$ & $23.0 \pm 1.5$ & $21.5 \pm 1.2$ & 0.53 & 0.38 \\
\hline \multirow[t]{2}{*}{$\mathrm{ALT}$} & IU/L & $5-45$ & & $24.8 \pm 3.8$ & $24.9 \pm 3.9$ & $23.8 \pm 2.8$ & $24.3 \pm 3.2$ & 0.56 & 0.81 \\
\hline & & $\mathrm{m} 80$ & $\mathrm{~m}$ & $47.2 \pm 8.7$ & $39.4 \pm 6.2$ & $47.7 \pm 9.0$ & $51.7 \pm 12.7$ & 0.88 & 0.46 \\
\hline \multirow[t]{2}{*}{$\gamma-\mathrm{GT}$} & IU/L & f 30 & $f$ & $27.7 \pm 5.2$ & $35.5 \pm 8.4$ & $30.0 \pm 5.5$ & $29.1 \pm 5.0$ & 0.43 & 0.58 \\
\hline & & & & $36.9 \pm 5.3$ & $38.4 \pm 5.2$ & $37.2 \pm 5.4$ & $39.8 \pm 6.9$ & 0.48 & 0.59 \\
\hline TP & $g / d L$ & $6.7-8.3$ & & $7.3 \pm 0.1$ & $7.3 \pm 0.1$ & $7.4 \pm 0.1$ & $7.4 \pm 0.1$ & 0.12 & 0.76 \\
\hline ALB & $d / d L$ & $3.8-5.3$ & & $4.5 \pm 0.1$ & $4.6 \pm 0.1$ & $4.6 \pm 0.1$ & $4.6 \pm 0.1$ & 0.06 & 0.74 \\
\hline hs-CRP & $\mathrm{mg} / \mathrm{dL}$ & 0.3 & & $0.10 \pm 0.0$ & $0 . I \pm 0.0$ & $0.1 \pm 0.0$ & $0.1 I \pm 0.1$ & 0.75 & 0.90 \\
\hline CRE & $\mathrm{mg} / \mathrm{dL}$ & $0.61-1.04$ & & $0.78 \pm 0.0$ & $0.8 \pm 0.0$ & $0.8 \pm 0.0$ & $0.79 \pm 0.0$ & 0.66 & 0.44 \\
\hline UA & $\mathrm{mg} / \mathrm{dL}$ & $3.8-7.0$ & & $5.5 \pm 0.2$ & $5.5 \pm 0.3$ & $5.5 \pm 0.2$ & $5.4 \pm 0.3$ & 0.89 & 0.85 \\
\hline UN & $\mathrm{mg} / \mathrm{dL}$ & $8-20$ & & $14.6 \pm 0.8$ & $13.5 \pm 0.5$ & $13.5 \pm 0.7$ & $14.5 \pm 0.7$ & 0.06 & 0.08 \\
\hline
\end{tabular}

$\mathrm{P}^{\mathrm{a}}$ values were calculated using paired $\mathrm{t}$ test. VLDL and LDL and HDL were evaluated using the Wilcoxon signed rank test, Active ${ }^{\mathrm{b}}$ : oyster extract supplement group, *: < 0.05, M, male; F, female;ALB, albumin;ALT, alanine aminotransferase; AST, aspartate aminotransferase; CRE, creatinine; $\gamma$-GT, $\gamma$-glutamyl transpeptidase; HDL, high-density lipoprotein; HDL-C, high density lipoprotein cholesterol; hs-CRP, high sensitivity c-reactive protein; LDL, low density lipoprotein; LDL-C, low density lipoprotein cholesterol;TG, triglyceride; Total-C, total cholesterol;TP, total protein; UA, uric acid; UN, urea nitrogen;VLDL, very low-density lipoprotein; SEM, standard error of means 
Table 4B Body composition data

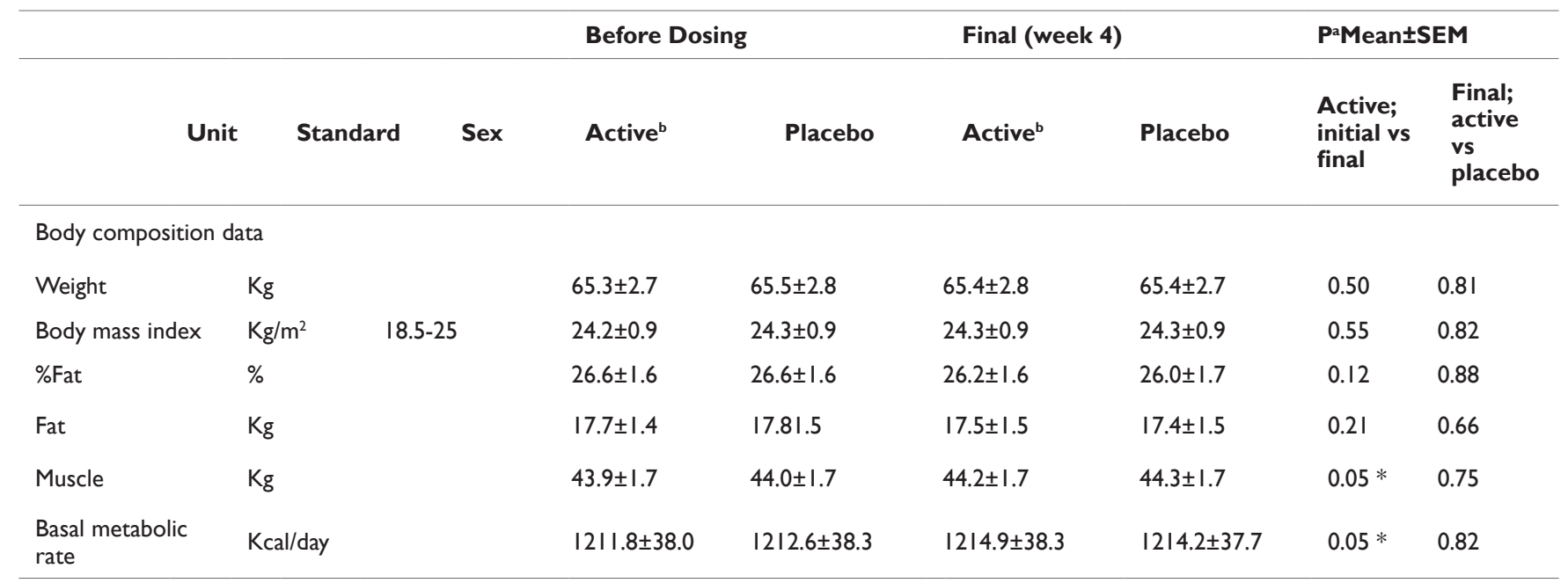

$\mathrm{Pa}^{\mathrm{a}}$ values were calculated using paired $\mathrm{t}$ test; Active ${ }^{\mathrm{b}}$, oyster extract supplement group; $*,<0.05, \mathrm{SEM}$, standard error of means

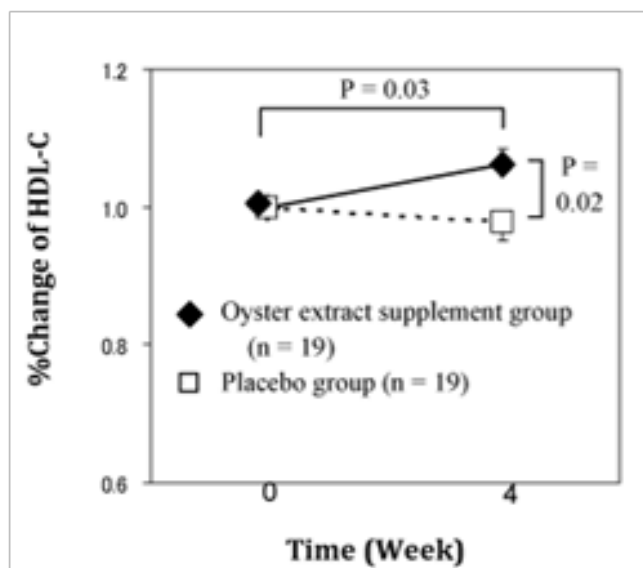

a: \%Change of HDL-C

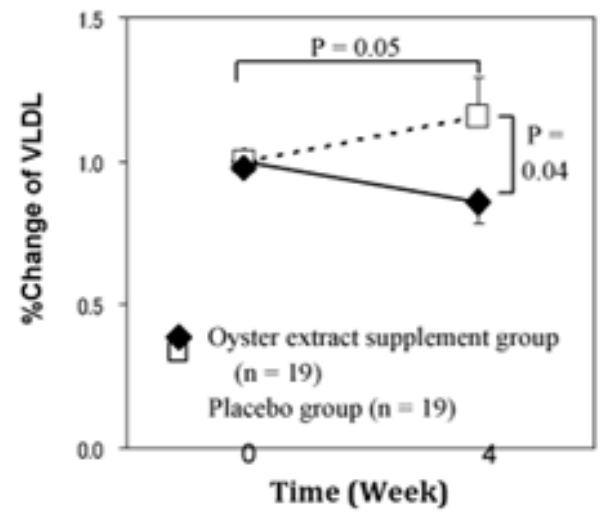

c: \%Change of VLDL

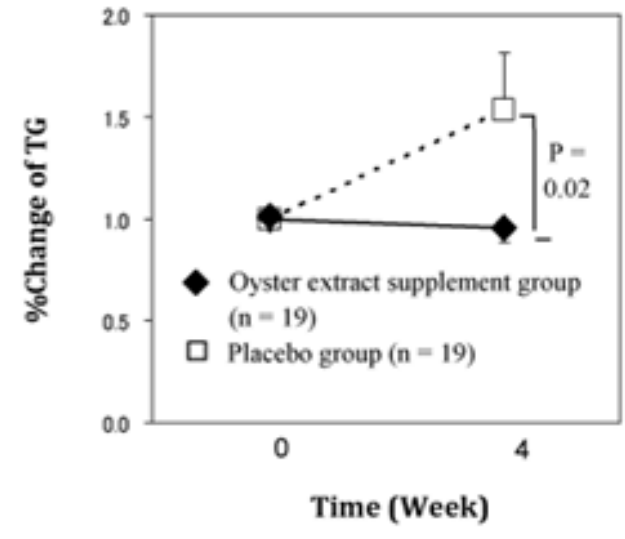

b: \%Change of TG

Figure $3 \%$ Changes of the means of HDL-C,TG, andVLDL over time in oyster extract supplement group or placebo group in the crossover, placebo-controlled, comparative study.

Two-sided $\mathrm{P}$ values were calculated for difference between the active ingredient and placebo groups in means using Mann-Whitney $U$ test. Two-sided $\mathrm{P}$ values were calculated for difference between the initial and final points using the Wilcoxon matched pairs signed rank test; Points, mean values; Bars, SEM.

Citation: Ohtani M, Seki T, Ooe M, et al. Effects of peptides and amino acids derived from oyster on blood lipids. J Nutr Health Food Eng. 20 I4; I (5): I66-I76. DOI: 10.15406/jnhfe.2014.01.00027 


\section{Body composition measurement in crossover placebo- controlled trial}

The body compositions listed in Materials and Methods were determined, the result showing no significant difference in most of items (data not shown) except the muscle mass and basal metabolisms between the groups of supplement and placebo. The muscle mass and basal metabolisms increased significantly in the supplement group though the change was not large (muscle mass; $\mathrm{P}=0.048$, basal metabolism; $\mathrm{P}=0.045$ ).

\section{Adverse events and side effects}

In the 2 trials, there was no subject who discontinued the trials, and there were no harmful effects with cause-and-effect relationship with the intake of the testing oyster supplement in the subject based on the serum chemistry and interviews.

\section{Study limitations}

In this report, the effects of the oyster supplement were validated in the open label study, followed by confirmation of the effect in the crossover placebo-controlled study.

As the result of the improvement of enzymatic hydrolysis conditions for oyster meat, the rate of low molecular fraction was increased in the compositions of the test supplement used in the crossover placebocontrolled study. In addition, since the number, age, and male/female ratio of the subjects were not same in the 2 trials, it was difficult to compare the results in the 2 studies. In the crossover placebocontrolled study, the decrease of HDL-C, and the increases of TG and VLDL were observed in the placebo group, while the tendency of increase of HDL-C, and the decreases of TG and VLDL was observed in the supplement group. Furthermore, the muscle mass and basal metabolisms in the supplement group significantly increased in the crossover trial. Hence, there was a concern that change of exercise habits and food habits interfered with the results. Based on these limitations, careful consideration is required for the interpretation of the present results.

\section{Discussion}

Before starting the present clinical trials, consent was obtained from medical workers who concerned about their blood lipid data, and the subjects with lipid abnormality in one or more parameters of blood lipid were sorted out in the screening.

The upper limit of BMI was to be $25 \mathrm{~kg} / \mathrm{m}^{2}$. In the open label trial, the subjects showed a mean BMI value of $25.3 \mathrm{~kg} / \mathrm{m}^{2}$ and were categorized into the obesity group. In the crossover trial, since the subjects showed a mean BMI value of $24.4 \mathrm{~kg} / \mathrm{m}^{2}$, they were categorized into non-obesity group (Table 1 ). In a diet survey, the average energy intake of subjects was of $2,234 \mathrm{kcal}$ in the open label trial. This value was not superfluous when compared with the energy requirement for the physical activity level III (male $2,800 \mathrm{kcal}$, female $2,200 \mathrm{kcal}$ ) to which workers who engage in jobs with frequent moving or standing are categorized. Therefore, as the cause(s) of dyslipidemia, the effects of the living habits including nutritional balances, exercise habits, or sleep habits may be predicted in addition to the food consumption.

In this study, we conducted the exploratory open label study at first to examine whether the lipid improvement effect observed in the test using animal models was reproduced or not in humans. The result of the open label study confirmed the improvement tendency of blood lipid values other than HDL-C at Week 8 in the 8 -week test duration.
Regarding lipoprotein compositions, we found the decrease of VLDL and the increase of HDL probably caused by the oyster supplement. The result indicates that the oyster meat supplement was likely to suppress the excessive production of VLDL, which is synthesized in the liver and plays a role to transport cholesterol into blood. In this trial, the ratios of VLDL decreased, while HDL ratio increased; this result was incompatible. Since there was a tendency that the values of TP, ALB, HDL-C, and LDL-C decreased at Week 4 but increased at Week 8 , we cannot exclude the changes of nutritional status and exercise quantity up to Week 4 from the placebo effects (Table 3 ).

Since we confirmed the effect of oyster meat supplement on the lipid metabolism in the open label study, we planned a 4week crossover placebo controlled comparison study to elucidate the effects of oyster meat supplement on HDL, HDL-C, and VLDL in which subjects with hyperlipidemia were included. The subjects received the oyster supplement tablets and the placebo tablets for 4weeks, respectively, while inserting a withdrawal period between the 2 administration durations. As a result, there observed significant changes of HDL-C $(\mathrm{P}=0.04)$, and $\operatorname{VLDL}(\mathrm{P}=0.03)$ after the administration of oyster meat supplement (Table 4). In other words, the oyster meat supplement group resulted in the suppression of HDL-C decrease and VLDL increase. The \%changes of the means of each parameter were compared over time in the two groups. Most of parameters did not show any difference in both groups, but HDLC, TG, and VLDL at Week 4 were significantly different from those of the placebo group with $\mathrm{P}=0.02,0.02$, and 0.04 , respectively (Figure 3 ).

The placebo tablets were prepared by replacing the oyster extract with maltitol of sugar alcohols (hydrogenated maltose starch). Maltitol is a low-calorie sweetener whose calorie is one half of sucrose. Energy per day of placebo tablet is $18 \mathrm{kcal}$, which is lower than the energy of the oyster supplement ( $21 \mathrm{kcal}$ per day). As the load of maltitol contained in the placebo tablets was small and approximately $2 \mathrm{~g}$ in a day, changes including the increase of TG due to the administration of maltitol could be neglected. Hence, the tendency of the decrease of HDL-C, the increase of TG, and the slight increase of VLDL observed in the placebo group (Table 4) was not attributable to the placebo tablets, but probably attributable to the changes of lifestyle habits of subjects. Since in the cross-over placebo-controlled trial, the same subjects received the oyster meat supplement and placebo under single blind conditions for the subjects, the changes of HDL-C, TG, and VLDL observed in the supplement group was thought to be the changes caused by the supplement per se. In the crossover placebocontrolled study, the increase of TG was inhibited; this result being compatible with the result in our animal model study. The oyster meat extract powder prepared by enzymatic degradation that was used in this study is reported to reduce the activities of fatty acid synthase and phosphoric acid glucose 6-dehydrogenase (G6PDH), and the amount of TG in the liver of Sprague-Dawley rats. ${ }^{11}$ Fatty acid synthetase synthesizes fatty acids from acetyl CoA, G6PDH converts glucose6-phosphoric acid to glycerol-3-phosphoric acid, and TG is formed by binding of the fatty acids with glycerol-3-phosphoric acid by the action of transferase. TG forms a complex with lipoproteins and generates VLDL, a precursor of LDL, which carries cholesterol.

The present 2 clinical trials confirm that the oyster meat supplement caused the decreases of the ratio of lipoprotein VLDL that carries TG. In the open label trial, lipoprotein VLDL decreased and LDL-C of blood lipid tended to decrease. In the crossover placebo controlled trial, VLDL decreased significantly, but LDL-C did not change. VLDL that is generated in the liver has a role to supply cholesterol to the 
peripheral tissues while changing to LDL. The cholesterol reduction effect of hydroxymethylglutaryl-CoA reductase inhibitors (statins) is attributed to their inhibitory activity on the biosynthesis of cholesterol. Different from such effect of statins, the active substance(s) in the oyster meat extract are likely to affect the formation of lipoproteins, which are a carrier of cholesterol, by inhibiting the VLDL synthesis through the control of hepatic TG synthesis. As shown in Table 4, in the cross-over placebo-controlled trial, lipoprotein HDL tended to increase $(\mathrm{P}=0.06)$, while blood lipid HDL-C significantly increased $(\mathrm{P}=0.04)$. HDL withdraws an excess amount of cholesterol from the peripheral tissues and inversely transports it to the liver in which cholesterol is subsequently converted to bile acids that are in turn removed by excretion into bile. In blood Apo A-I enters peripheral cells, withdraws cholesterol, returns to the circulation, and converts to HDL. HDL matures cholesterol by esterification. Cholesterol esters in HDL are exchanged with TG from VLDL or LDL through cholesteryl ester transfer protein (CETP). It is known that HDL is increased when CETP is inhibited. ${ }^{12}$ CETP inhibitors have been being expected as new lipid-modifying drugs that increase HDL, and among the food constituents, polyphenols in hops are reported to inhibit CETP. ${ }^{13}$ Since oyster meat supplement obtained by the enzyme degradation resulted in the reduction of VLDL and the increase of HDL, one or more functional amino-acid and peptide components having these functions will be identified in the future.

Regarding degradation products by enzyme digestion from the food proteins, peptides (remaining as the indigestible state) originated from soy proteins have bile acid-binding capacity, and reduce cholesterol, a precursor of bile acids. ${ }^{14}$ Peptides contained in the oyster meat extracts were unlikely to inhibit the absorption of bile acids or TG in the digestive tract; because the peptides were predicted to be dipeptides based on the molecular weight distribution. As the low molecular weight peptides which are known as active components to reduce TG, valyl threonyl leucine, valyl tyrosyl proline, and valyl tyrosyl proline are present in pig erythrocyte hemoglobin hydrolysate. ${ }^{8}$

Transcription factor SREBP (sterol regulatory element-binding protein) regulates metabolism of fatty acids, TG, and cholesterol. In animal obesity models, fatty acid synthesis was accelerated by SREBP activation. SREBP is thought to be controlled by cholesterol contents in a body mainly, but Inoue et al. report that it is also controlled by glutamine, one of amino acids..$^{15}$ Taurine, which is a sulfur-containing amino acid and is contained in oyster meat extracts, has a cholesterol reduction efficacy. Cholesterol that is transported to the liver by HDL is catabolized to bile acids. Then, the acids are conjugated with Taurine, and the conjugates are excreted into the intestinal tract via bile. In recent years, Taurine is known to be secreted from adipose cells and is expected as a novel adipocytokine which improves the inhibition of expression of adiponectin due to obesity. ${ }^{16}$

In this study the muscle mass and basal metabolic rate were low but significantly ( $\mathrm{P}=0.05$, respectively) increased at Week 4 in the supplement group of the crossover placebo-controlled study (Table 4); the reason for this is probably ascribable to the elevation of activity caused by the improvement of nutritional state by supplementation of amino acids and peptides. Ohtani et al., ${ }^{4}$ report that the nutritional state was improved in their clinical study in elder person aged at 83 (average) who received $1,800 \mathrm{mg}$ /day of amino acids for a long duration.

Since the number of examinees, age, and the ratio of male and female subjects were different in the present 2 trials, it is difficult to exactly compare the results of 2 studies. However, since the improvement of enzymatic degradation in the crossover trial resulted in the change of components in the oyster extract as described above, there is a possibility that the change in the quality and/or amounts of peptides may affect the data in the crossover trial.

From now on, we will identify the functional amino acids and peptides contained in the oyster meat extract and elucidate their mechanism of action, and thereby we will develop the safer and more effective functional foods for the purpose of the primary prevention of lifestyle-related diseases.

\section{Acknowledgements}

The authors would like to thank Fumishige HINAMI, MD, Kouji SAWADA, MD, Toyohiko TSUKANO, MD, Jun-ya OMOTAKA, and Takashi ICHIKAWA (Sansei Hospital, Sansei-kai Medical Treatment Corporation) for their support. We also thank Hidenobu Okumura, Seiji KURITA, and Hiroshi MATSUI (Noevir Co., Ltd.), and Hiroshi Okumura, Masahiko Ooe and Kosumo KOUNO (Tokiwa Pharmaceutical Co., Ltd.) for their assistance.

\section{Conflict of interest}

Author declares that there is no conflict of interest.

\section{References}

1. Tanabe N, Iso H, Okada K, et al. Serum total and non-high-density lipoprotein cholesterol and the risk prediction of cardiovascular events the JALS-ECC. Circ J. 2010;74(7):1346-1356.

2. Japan Atherosclerosis Society. Japan Atherosclerosis Society (JAS) guidelines for prevention of atherosclerotic cardiovascular diseases. $J$ Atheroscler Thromb. 2007:5-57.

3. Statistics Department of Ministry of Agriculture, Forestry and Fisheries of Japan. Annual statistics of fishery and fish culture [article in Japanese]. 2012.

4. Ohtani M, Watanabe M, Ueda J, et al. Nutritional effect of supplementation of energy jelly with amino acid for elder patient in long-term hospitalization [article in Japanese]. J Jap Soc Clin Nut. 2009;30:330-338.

5. Murakami S, Kurihara S, Koikawa N, etal. Effects of oral supplementation with cystine and theanine on immune function of athletes in endurance exercise at a training camp: randomized, double-blind, placebocontrolled trial. Biosci Biotechnol Biochem. 2009;73(4):817-821.

6. Kawada S, Kobayashi K, Ohtani M, et al. Cystine and theanine supplementation restores high-intensity resistance exercised-induced attenuation of natural killer cell activity in well-trained men. $J$ Strength Cond Res. 2010;24(3):846-851.

7. Murakami S, Kurihara S, Titchenal CA, et al. Suppression of exerciseinduced neutrophilia and lymphopenia in athletes by cystine/theanine intake: a randomized, double-blind, placebo-controlled trial. J Int Soc Sports Nutr. 2001;7(1):23.

8. Kagawa K, Matsutaka H, Fukuhama C, et al. Globin digest, acidic protease hydrolysate, inhibits dietary hypertriglyceridemia and Val-ValTyr-Pro, one of its constituents, possesses most superior effect. Life Sci. 1996;58(20):1745-1755.

9. Shiozaki K, Shiozaki M, Masuda J, et al. Identification of oyster-derived hypotensive peptide acting as angiotensin I converting enzyme inhibitor. Fish Sci. 2010;76:865-872.

10. Lee TG, Maruyama S. Isolation of HIV-1 protease-inhibiting peptides from thermolysin hydrolysate of oyster proteins. Biochem Biophys Res Commun. 1998;253(3):604-608. 
11. Tanaka K, Nishizono S, Kugino K, et al. Effects of dietary oyster extract on lipid metabolism, blood pressure, and blood glucose in SD rats, hypertensive rats, and diabetic rats. Biosci Biotechnol Biochem. 2006;70(2):462-470.

12. Li C, Zhang W, Zhou F, et al. Cholesteryl ester transfer protein inhibitors in the treatment of dyslipidemia: a systematic review and meta-analysis. PLoS One. 2013;8(10):e77049.

13. Hirata H, Yimin, Segawa S, et al. Xanthohumol prevents atherosclerosis by reducing arterial cholesterol content via CETP and apolipoprotein E in CETP-transgenic mice. PLoS One. 2012;7(11):e49415.
14. Nagaoka S, Nakamura A, Shibata $H$, et al. Soystatin (VAWWMY), a novel bile acid-binding peptide, decreased micellar solubility and inhibited cholesterol absorption in rats. Biosci Biotechnol Biochem. 2010;74(8):1738-1741.

15. Inoue $\mathrm{J}$, Ito $\mathrm{Y}$, Shimada $\mathrm{S}$, et al. Glutamine stimulates the gene expression and processing of sterol regulatory element binding proteins, thereby increasing the expression of their target genes. FEBS J. 2011;278(15):2739-2750.

16. Tsuboyama-Kasaoka N, Shozawa C, Sano K, et al. Taurine (2-aminoethanesulfonic acid) deficiency creates a vicious circle promoting obesity. Endocrinology. 2006;147(7):3276-3284. 